\title{
Fatores associados ao estado nutricional de crianças menores de cinco anos da Paraíba, Brasil
}

\author{
Factors associated with nutritional status of children under five \\ years old from Paraíba, Brazil
}

Maria Mônica de Oliveira (https://orcid.org/0000-0002-2775-4318) ${ }^{1}$

Eduarda Emanuela Silva dos Santos (https://orcid.org/0000-0002-3369-8930) ${ }^{1}$

Ítalo de Macedo Bernardino (https://orcid.org/0000-0003-4750-5666) ${ }^{1}$

Dixis Figueroa Pedraza (http://orcid.org/0000-0002-5394-828X) ${ }^{1}$
${ }^{1}$ Universidade Estadual da Paraíba. R. das Baraúnas 351, Campus Universitário, Bodocongó. 58109-753 Campina Grande PB Brasil. dixisfigueroa@gmail.com

\begin{abstract}
This paper aimed to analyze whether the nutritional status of children under 5 years of age is related to the biological conditions of their mothers, access to health services, the benefit of social programs, and socioeconomic conditions. This is a cross-sectional study carried out in the context of the Family Health Strategy in seven municipalities in inland Paraíba State. The methodology included the diagnosis of stunting and excess weight (overweight and obesity) in children, which determinants were analyzed through the Decision Tree. As a result, 469 children were evaluated, of which $7.9 \%$ had stunting and $12.8 \%$ had excess weight. An association of these outcomes was found with maternal nutritional status. Also, the relevant exposures of stunting were the age of the child of less than 2 years $(p=0.018)$ and the moderate/severe food and nutritional insecurity $(p=0.008)$. For excess weight, not being a beneficiary of the Programa Bolsa Familia $(p=0.049)$ and the worst socioeconomic situation ( $p=0.006$ ) were also factors associated with the outcome. As a conclusion of the present study, we can affirm that there is an association between the maternal and child nutritional status.
\end{abstract}

Key words Nutritional Status, Overweight, Growth, Family Health Strategy, Child
Resumo Objetivou-se analisar se o estado nutricional de crianças menores de cinco anos de idade está relacionado às condições biológicas de suas mães, ao acesso a serviços de saúde, ao benefício de programas sociais e às condições socioeconômicas. Trata-se de um estudo transversal realizado no contexto da Estratégia Saúde da Família, em sete municípios do interior do estado da Paraíba. A metodologia incluiu o diagnóstico do déficit de estatura e do excesso de peso (sobrepeso e obesidade) das crianças, cujos determinantes foram analisados por meio de árvore de decisão. Como resultado, foram avaliadas 469 crianças, das quais 7,9\% apresentaram déficit de estatura e 12,8\% excesso de peso. Encontrou-se associação desses desfechos com o estado nutricional materno. A baixa estatura também teve como exposições relevantes a idade da criança inferior a dois anos $(p=0,018)$ $e$ a insegurança alimentar e nutricional moderada/grave $(p=0,008)$. Para o excesso de peso, não ser beneficiário do Programa Bolsa Família ( $p=0,049)$ e a pior situação socioeconômica ( $p=$ $0,006)$ também representaram fatores associados ao desfecho. Como conclusão do presente estudo, podemos afirmar que existe uma associação entre o estado nutricional materno e o da criança.

Palavras-chave Estado nutricional, Sobrepeso, Crescimento, Estratégia Saúde Família, Criança 


\section{Introdução}

Estados nutricionais extremos de déficits e excessos constituem importantes problemas de saúde pública que tendem a ser graves entre mulheres em idade reprodutiva e crianças menores de cinco anos. Essas condições afetam negativamente as funções reprodutivas das mulheres e estão associadas a morbi-mortalidade, desenvolvimento cognitivo e crescimento da criança, bem como prejudicam a produtividade econômica ${ }^{1,2}$. Metas globais foram propostas para melhorar a nutrição materna e infantil até 2025 , reconhecendo-se a necessidade de resolver o problema generalizado da dupla carga de desnutrição ${ }^{3}$. A esse propósito se conectam os objetivos de desenvolvimento sustentável, que em uma de suas metas visa acabar com todas as formas de má nutrição até $2030^{4}$.

O atual cenário do estado nutricional da população brasileira, caracterizado pelo processo de transição nutricional, reflete as mudanças vivenciadas no perfil demográfico e epidemiológico do país ao longo das últimas três décadas ${ }^{5}$. Embora se evidencie que a prevalência global da desnutrição esteja diminuindo tanto em crianças quanto em mulheres, nos países de baixa e média renda, como o Brasil, observa-se a coexistência da baixa estatura com o sobrepeso e/ou obesidade ${ }^{1,2}$, que pode acontecer no mesmo país ou cidade, na mesma família (binômio mãe/filho) ou no mesmo indivíduo em diferentes estágios de sua vida ${ }^{6}$.

No Brasil, a prevalência de excesso de peso estimada por meio da pesquisa Vigitel de 2019 para a população feminina adulta residente nas capitais dos estados e no Distrito Federal foi de $53,9 \%{ }^{7}$. Essa proporção equivale à reportada em nível mundial entre os países mais afetados pelo problema ${ }^{8}$. Para os menores de cinco anos, a prevalência do excesso de peso no Brasil foi de 7,3\% em $2006^{9}$, enquanto mundialmente é estimada em $5,6 \%{ }^{10}$. Em relação ao déficit de estatura, as prevalências são mais distantes: 7,1\% no caso das crianças brasileiras $^{9}$ e 22,2\% globalmente ${ }^{10}$.

$\mathrm{Na}$ etiologia desses problemas, destaca-se a influência da interação de fatores biológicos, ambientais, comportamentais, sociais, econômicos e políticos. Assim, o estado nutricional infantil resulta da sinergia de determinantes que incluem o estado nutricional materno, o peso ao nascer, o estado de saúde e as práticas alimentares da criança, os cuidados com a criança, a segurança alimentar e nutricional (SAN) da família, o acesso aos serviços de saúde e o status socioeconômico ${ }^{1,11}$. Nesse contexto, o ambiente familiar se sobressai como meio no qual os múltiplos fatores de risco podem se manifestar e influenciar a situação de saúde ${ }^{11,12}$, com as características maternas representando uma importante interface entre a criança e o meio ambiente ${ }^{13}$. Além disso, não se deve descartar a possibilidade de influência dos cuidados prestados pelos serviços de saúde no estado nutricional, como constatado em uma revisão da literatura que apontou maiores chances de déficit de estatura entre crianças usuárias de serviços públicos de saúde ou de programas sociais $^{14}$.

Apesar de vasta literatura sobre a temática, a compreensão da etiologia do estado nutricional das crianças brasileiras ainda precisa de ênfases relacionadas à SAN e nos cuidados de saúde, inclusive da influência do Programa Bolsa Família $(\mathrm{PBF})^{14,15}$. Na perspectiva da assistência à saúde, recomenda-se a necessidade de estudos com foco no desempenho das equipes do Programa Mais Médicos (PMM) em relação às convencionais ${ }^{16}$.

$\mathrm{O}$ objetivo desse trabalho foi analisar se o estado nutricional de crianças menores de cinco anos de idade está relacionado às condições biológicas de suas mães, ao acesso a serviços de saúde, ao benefício de programas sociais e às condições socioeconômicas.

\section{Métodos}

\section{Desenho do estudo}

Trata-se de um estudo transversal com crianças menores de cinco anos de idade. A pesquisa foi desenvolvida em 2018.

\section{Contexto}

Foram selecionadas para observação municípios do estado com população entre 30.000 e 149.999 habitantes que recebem incentivos de custeio para a implementação de ações de prevenção e controle do sobrepeso em crianças ${ }^{17}$. Do total de 12 municípios com esse benefício, um foi excluído por não possuir cobertura total da ESF, e outro por não ter no sistema de saúde equipes da ESF predeterminadas como de interesse para o estudo (equipes convencionais vinculadas a Núcleo de Apoio à Saúde da Família sem a inclusão de nutricionista e equipes do PMM vinculadas a Núcleo de Apoio à Saúde da Família com a atuação de nutricionista). Além disso, três municípios não fizeram parte do estudo devido à sua inserção em outra proposta com caracterís- 
ticas similares. Em cada município foram incluídas todas as equipes de saúde com os critérios de seleção, totalizando 22 estabelecimentos (11 convencionais e a mesma quantidade do PMM), e uma escola de educação infantil, selecionada por sorteio aleatório simples, por equipe de saúde participante do Programa Saúde na Escola, totalizando 11 instituições.

Considerou-se como população de estudo as crianças de 0 a 59 meses de idade residentes em áreas urbanas dos municípios do interior da $\mathrm{Pa}$ raíba selecionados para participar da pesquisa e usuárias da ESF. Por terem particularidades relacionadas ao estado nutricional, crianças gêmeas, adotadas e de mães com idade inferior a 18 anos foram excluídas.

O tamanho da amostra foi definido considerando $\mathrm{N}=23.089, \mathrm{p}=18,7 \%$ (proporção de menores de cinco anos com excesso de peso nos municípios de estudo em 2017 segundo dados do SISVAN: http://dabsistemas.saude.gov.br/ sistemas/sisvanV2/relatoriopublico/index), erro amostral máximo de 4\% sob nível de confiança de $95 \%$ e $25 \%$ para o controle de perdas e confundimento, estimando-se a necessidade de observar 479 indivíduos. Essa amostra também é suficiente para o déficit de estatura, desfecho para o qual haveria a necesidade de estudar 226 crianças, considerando prevalência esperada de 7,7\%, com base no SISVAN, e as mesmas características de cálculo estabelecidas para o excesso de peso. A quantidade de crianças por município foi estabelecida de forma proporcional. A seleção das crianças foi por sorteio aleatório simples, com base nos registros das equipes de saúde e escolas de educação infantil. O fluxograma do desenho de seleção da amostra de estudo está disponivel na Figura 1.

\section{Dados coletados}

Os entrevistadores aplicaram individualmente, em local apropriado, um questionário estruturado às mães das crianças, e antropometristas treinados realizaram a avaliação antropométrica das crianças/mães. As informações foram organizadas em blocos: condições biológicas das mães/ crianças ( sexo da criança, idade da criança, idade da mãe, estatura da mãe, peso da mãe), acesso a serviços de saúde (trimestre de início do pré-natal, suplementação de vitamina A no pós-parto, tipo de equipe de saúde da ESF), condições socioeconômicas (trabalho materno, convivência da mãe com companheiro, SAN, classificação socioeconômica) e benefício de programas so- ciais (frequência na escola de educação infantil, benefício do PBF).

As crianças e suas mães foram medidas (comprimento em menores de 24 meses e estatura para crianças de 25-60 meses e mães) e pesadas com equipamentos e técnicas padronizados ${ }^{18}$. O comprimento foi aferido utilizando antropômetro infantil de madeira com amplitude de $130 \mathrm{~cm} \mathrm{e}$ subdivisões de $0,1 \mathrm{~cm}$ (posição deitada). A estatura foi medida usando estadiômetro (WCS) com escala em milímetros ( $\mathrm{mm}$ ) (posição em pé e descalço). Para o peso, utilizou-se balança eletrônica do tipo plataforma com capacidade para $150 \mathrm{~kg}$ e graduação em 100g (Tanita UM-080) (descalço e com uso de peças leves). Todas as medidas foram realizadas duas vezes e a média foi utilizada para fins de registro.

Os escores-Z de estatura-para-idade (E/I) e Índice de Massa Corporal-para-idade (IMC/I) das crianças foram calculados com o uso do software WHO Anthro v.3, considerando como referência a população do Multicentre Growth Reference Study $^{19}$. Crianças com escores- $Z<-2$ de E/I foram classificadas com déficit de estatura. Crianças com Escores-Z > 2 de IMC/I foram consideradas com excesso de peso (sobrepeso e obesidade) ${ }^{18}$.

A baixa estatura materna foi definida pelo ponto de corte $155,0 \mathrm{~cm}$, que corresponde ao percentil cinco da relação estatura/idade em $\geq 20$ anos, segundo o National Center for Health Statistic (CDC Growth Charts. http://www.cdc.gov/ growthcharts/cdc_charts.htm, acessado em 18 de setembro de 2018). O Índice de Massa Corporal (IMC) da mãe foi calculado pela razão entre o peso $(\mathrm{kg})$ e a estatura (metros) ao quadrado. Para diagnóstico, utilizaram-se os seguintes pontos de corte de IMC: baixo peso $(<18,5)$, peso adequado $(18,5-24,9)$ e sobrepeso e obesidade $(\geq 25)^{18}$.

Como parte do questionário, a situação da SAN das famílias foi estimada com base na Escala Brasileira de Insegurança Alimentar, com 14 itens, que classifica as famílias em segurança alimentar, insegurança alimentar leve, insegurança alimentar moderada e insegurança alimentar grave ${ }^{20}$. A SAN familiar foi dicotomizada em segurança alimentar/insegurança alimentar leve e insegurança alimentar moderada/grave.

A classificação socioeconômica da família se baseou nos critérios da Associação Brasileira de Empresas de Pesquisa ${ }^{21}$. Para essa classificação, considera-se a existência de vaso sanitário no domicílio, a contratação de empregada doméstica, a posse de bens, o grau de instrução do chefe de família e o acesso a serviços públicos. As famílias foram classificadas nas classes $\mathrm{A} / \mathrm{B} / \mathrm{C}$ ou $\mathrm{D} / \mathrm{E}$. 
23.089 menores de cinco anos de sete municípios com incentivos de custeio para a implementação de ações de prevenção e controle do sobrepeso em crianças

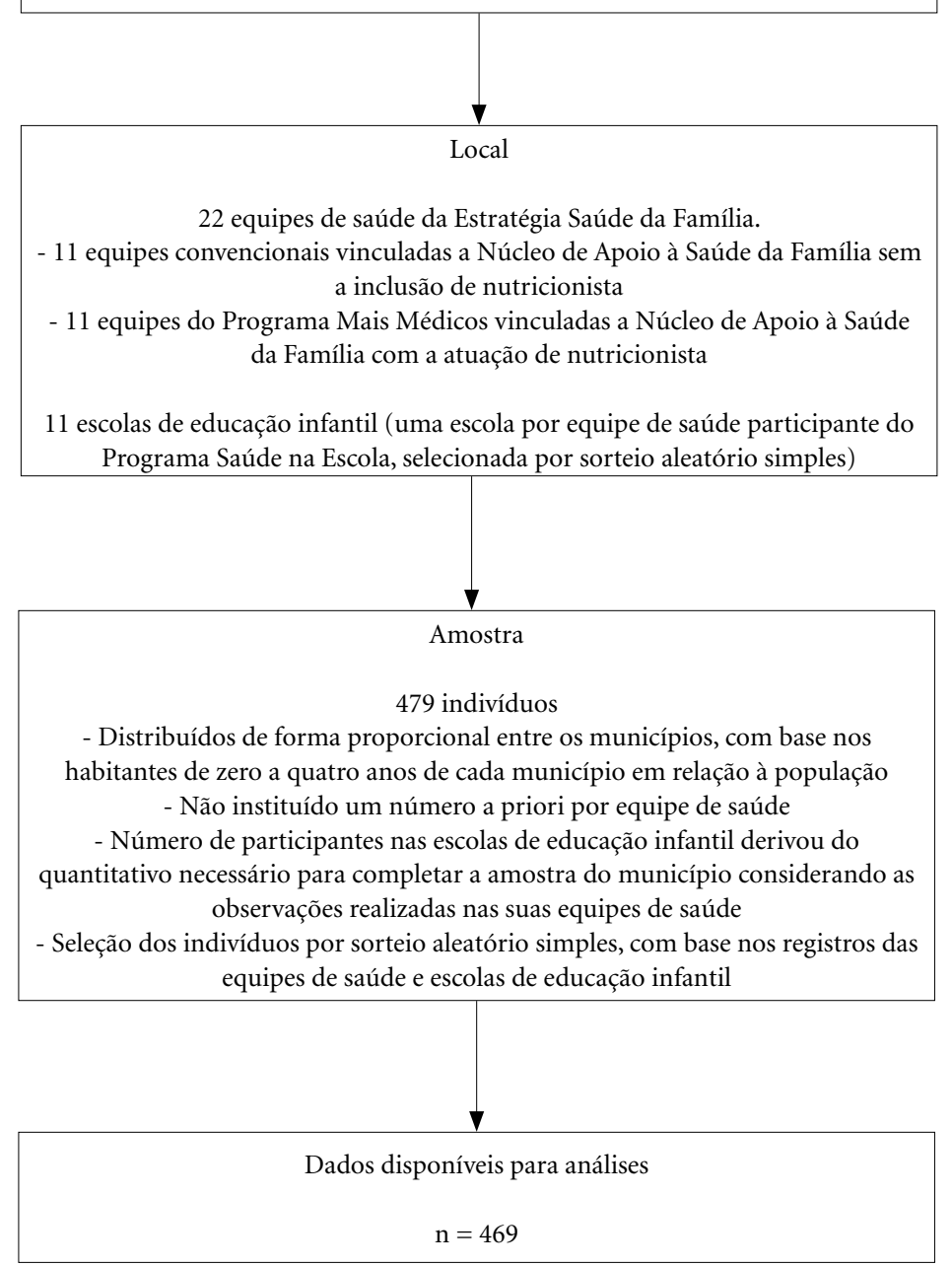

Figura 1. Fluxograma do desenho de seleção da amostra de estudo para análise de associação do estado nutricional de crianças menores de cinco anos com características materno-infantis em municípios do interior do estado da Paraíba, 2018.

Fonte: Autores.

O grupo de variáveis independentes foi formado pelas seguintes características: idade da criança $(\geq 2 ;<2$ anos), sexo da criança (masculino; feminino), idade materna ( $\geq 20 ;<20$ anos), estatura da mãe (adequada; inadequada), IMC da mãe (adequado; sobrepeso e obesidade), trimestre de início do pré-natal ( $1^{\circ}$ trimestre de gestação; após $1^{\circ}$ trimestre de gestação), suplementação de vitamina A no pós-parto (sim; não), tipo de equipe de saúde da ESF (PMM; convencional), trabalho ma- terno (sim; não), convivência da mãe com companheiro (sim; não), SAN (segurança alimentar/ insegurança alimentar leve; insegurança alimentar moderada/grave), classificação socioeconômica (classes A/B/C; classes D/E), frequência da criança à escola de educação infantil (sim; não), benefício do PBF (sim; não). O déficit de estatura e o excesso de peso (sobrepeso e obesidade) das crianças representaram as variáveis dependentes. O modelo conceitual proposto encontra-se na Figura 2. 


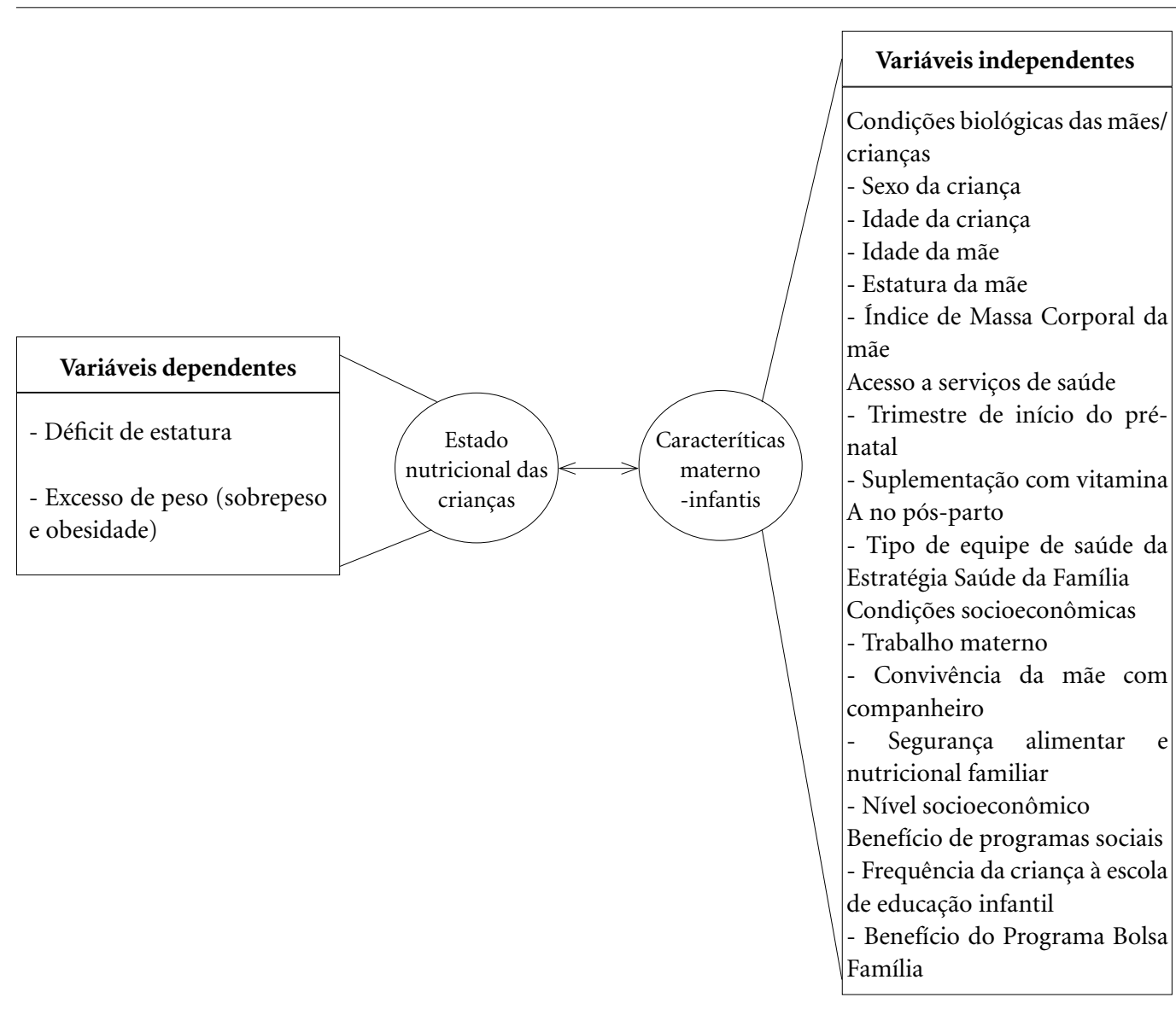

Figura 2. Modelo conceitual da análise de associação do estado nutricional de crianças menores de 5 anos com características materno-infantis em municípios do interior do estado da Paraíba, 2018.

Fonte: Autores.

\section{Análise estatística}

Inicialmente, foram calculados as frequências absolutas e os percentuais de todas as variáveis em estudo. Em seguida, utilizando o teste $\chi^{2}$ ou o teste exato de Fisher, foi realizada a análise bivariada para testar a associação entre o déficit de estatura e o excesso de peso da criança com as variáveis independentes.

Para explicar o déficit de estatura e o excesso de peso das crianças, foram inseridas todas as variáveis em modelos de árvore de decisão por meio do algoritmo $\mathrm{CHAID}^{22}$. Esse método consiste em regras de decisão que realizam sucessivas divisões no conjunto de dados, de modo a torná-lo cada vez mais homogêneo com relação à variável dependente. A árvore de decisão utiliza um gráfico que começa com um nó raiz em que todas as observações da amostra são apresentadas. Os nós produzidos em sequência representam subdivi- sões dos dados em grupos cada vez mais homogêneos, sendo denominados nós-filhos. Quando não há mais possibilidade de divisão, os nós são chamados de nós-terminais ou folhas ${ }^{22,23}$. O modelo foi ajustado mediante sucessivas divisões binárias (nós) nos conjuntos de dados. O critério de parada adotado foi o valor $\mathrm{p}<0,05$ da estatística $\chi^{2}$ usando a correção de Bonferroni. $\mathrm{O}$ ajuste do modelo final foi avaliado pela estimativa de risco geral, que compara a diferença entre o valor esperado e o observado pelo modelo, indicando em que medida a árvore corretamente prediz os resultados. Só permaneceram nos gráficos finais as variáveis que obtiveram valor $\mathrm{p}<0,05$.

\section{Aspectos éticos}

O projeto de pesquisa foi aprovado pelo Comitê de Ética em Pesquisa da Universidade Estadual da Paraíba. Todos os participantes da 
pesquisa assinaram o Termo de Consentimento Livre e Esclarecido. Pautado em aspectos éticos, no final da coleta dos dados, um município no qual os gestores inicialmente se recusaram a participar da pesquisa foi reincluído por solicitação deles próprios. Dessa forma, as perdas na coleta dos dados foram compensadas no município em questão, preservando-se o tamanho da amostra desenhado para a pesquisa.

\section{Resultados}

Das 479 crianças que participaram do estudo, foram analisados os dados das 469 que tiveram avaliação antropométrica completa. Para o caso do excesso de peso, oito casos com déficit de peso fora excluídos das análises. As prevalências de déficit de estatura e excesso de peso foram 7,9\% (IC 95\%: 6,1-10,0) e 12,8\% (IC 95\%: 10,1-15,3), respectivamente.

Do total de crianças avaliadas, observa-se que $217(46,3 \%)$ eram menores de dois anos e $243(51,8 \%)$ do sexo masculino. Quanto ao estado nutricional materno, evidenciou-se baixa estatura de 35,4\% (IC 95\%: 31,1-37,9) e sobrepeso e obesidade de 59,7\% (IC 95\%: 56,5-63,2). A maioria das mães $(80,6 \%)$ relatou ter iniciado o pré-natal no $1^{\circ}$ trimestre de gestação e apenas $39,8 \%$ informam ter sido suplementadas com vitamina A no pós-parto. Das famílias, $17,5 \%$ estavam em insegurança alimentar moderada/grave, $64,0 \%$ pertenciam às classes socioeconômicas D/E e 73,8\% recebiam benefício do PBF. A frequência da criança na escola de educação infantil girou em torno de 50\% (Tabela 1).

Segundo análises bivariadas, ser menor de dois anos de idade $(\mathrm{p}=0,018)$ foi a única condição associada ao déficit de estatura (Tabela 1). O início do pré-natal após o $1^{\circ}$ trimestre de gestação $(\mathrm{p}=0,023)$ e não ser beneficiário do PBF ( $\mathrm{p}$ $=0,049$ ) se associaram ao excesso de peso (Tabela 2).

Por meio do modelo final da árvore de decisão para o déficit de estatura, foi possível constatar que, além da idade da criança, a SAN e a estatura materna influenciaram os resultados. $\mathrm{O}$ modelo ficou constituído por sete nós, tendo a idade da criança como primeiro nó-filho, com ramificação apenas para as menores de dois anos, que foram as que apresentaram maior prevalência do desfecho $(p=0,018)$. Nessas crianças, a insegurança alimentar moderada/grave exerceu importante influência na prevalência de baixa estatura $(p=0,008)$. Nas crianças de famílias em segurança alimentar/insegurança alimentar leve, a baixa estatura foi mais acentuada quando a mãe teve o mesmo diagnóstico $(\mathrm{p}=0,028)$ (Figura 3 ).

Para o excesso de peso, o modelo final da árvore de decisão apresentou nove nós. Segundo o modelo, não ser beneficiário do PBF foi a condição que mais influenciou o desfecho ( $\mathrm{p}=$ 0,049 ), com prevalência de $17,9 \%$, enquanto nos beneficiários do Programa foi de 10,9\%. Entre as crianças não beneficiárias do $\mathrm{PBF}$, ser das classes socioeconômicas $\mathrm{D} / \mathrm{E}$ representou uma prevalência de excesso de peso quase três vezes maior em comparação às crianças das classes $\mathrm{A} / \mathrm{B} / \mathrm{C}(\mathrm{p}$ $=0,006)$. Na sequência da situação socioeconômica, como nós-terminais, observa-se que nas crianças das classes $\mathrm{A} / \mathrm{B} / \mathrm{C}$ o excesso de peso foi quase sete vezes maior nas crianças de mães com a mesma classificação ( $\mathrm{p}=0,027)$; nas crianças das classes socioeconômicas $\mathrm{D} / \mathrm{E}$, a baixa estatura materna influenciou a presença de excesso de peso em seus filhos $(\mathrm{p}=0,019)$, que tiveram prevalência de $53,3 \%$ versus $18,5 \%$ na condição contrária da mãe. O trimestre de início do prénatal perdeu a significância estatística após ajuste do modelo pelo nível socioeconômico (Figura 4).

\section{Discussão}

O estudo em questão avaliou fatores associados a desvios nutricionais em crianças em vulnerabilidade assistidas pela ESF. Nesse sentido, destacaram-se prevalências expressivas de déficit de estatura e de excesso de peso associadas ao estado nutricional materno. Contudo, o estado nutricional da criança não apresentou associação com o tipo de equipe de saúde, o que talvez reflita padrões semelhantes no acesso aos serviços de saúde e a multiplicidade de fatores envolvidos na implantação das ações de cuidado nutricional ${ }^{14,24}$.

A situação nutricional evidenciada no presente estudo revela um quadro epidemiológico de relevância em saúde pública, caracterizado pela coexistência de déficit de estatura e sobrepeso e obesidade entre crianças menores de cinco anos, refletindo a realidade de países da América Latina ${ }^{25}$ e do Brasil ${ }^{26}$. Esse panorama também representa a situação das crianças brasileiras que frequentam as unidades básicas de saúde ou que fazem parte de cadastros sociais, cujas prevalências de déficit de estatura $(7,3 \%)$ e de sobrepeso/ obesidade $(11,0 \%)$ estimadas em metanálise ${ }^{14}$ são semelhantes às encontradas. 
Tabela 1. Estado nutricional expresso em escore z da estatura-para-idade, de acordo com condições biológicas das mães/crianças, acesso a serviços de saúde, benefício de programas sociais e condições socioeconômicas, de crianças menores de cinco anos residentes em municípios do interior do estado da Paraíba, 2018.

\begin{tabular}{|c|c|c|c|c|c|c|c|}
\hline \multirow{3}{*}{ Variáveis } & \multicolumn{6}{|c|}{ Estatura-para-idade (escore $\mathrm{z}$ ) } & \multirow{3}{*}{ p-valor } \\
\hline & \multicolumn{2}{|c|}{ Total } & \multicolumn{2}{|c|}{$\geq-2$} & \multicolumn{2}{|c|}{$<-2$} & \\
\hline & $\mathbf{n}$ & $\%$ & $\mathbf{n}$ & $\%$ & $\mathbf{n}$ & $\%$ & \\
\hline \multicolumn{8}{|l|}{ Condições biológicas das mães/crianças } \\
\hline Idade da criança (anos) & & & & & & & 0,018 \\
\hline$\geq 2$ & 252 & 53,7 & 239 & 94,8 & 13 & 5,2 & \\
\hline$<2$ & 217 & 46,3 & 193 & 88,9 & 24 & 11,1 & \\
\hline Sexo da criança & & & & & & & 0,332 \\
\hline Masculino & 243 & 51,8 & 221 & 90,9 & 22 & 9,1 & \\
\hline Feminino & 226 & 48,2 & 211 & 93,4 & 15 & 6,6 & \\
\hline Idade materna (anos) & & & & & & & 0,562 \\
\hline$\geq 20$ & 420 & 89,9 & 386 & 91,9 & 34 & 8,1 & \\
\hline$<20$ & 47 & 10,1 & 45 & 95,7 & 2 & 4,3 & \\
\hline Estatura da mãe $(\mathrm{cm})$ & & & & & & & 0,079 \\
\hline Adequada & 303 & 64,6 & 284 & 93,7 & 19 & 6,3 & \\
\hline Inadequada $(<155)$ & 166 & 35,4 & 148 & 89,2 & 18 & 10,8 & \\
\hline Índice de Massa Corporal da mãe $\left(\mathrm{Kg} / \mathrm{m}^{2}\right)$ & & & & & & & 0,076 \\
\hline Adequado & 189 & 40,3 & 169 & 89,4 & 20 & 10,6 & \\
\hline Sobrepeso e obesidade $(\geq 25)$ & 280 & 59,7 & 263 & 93,9 & 17 & 6,1 & \\
\hline \multicolumn{8}{|l|}{ Acesso a serviços de saúde } \\
\hline Trimestre de início do pré-natal & & & & & & & 0,933 \\
\hline $1^{\circ}$ trimestre de gestação & 377 & 80,6 & 347 & 92,0 & 30 & 8,0 & \\
\hline Após $1^{\circ}$ trimestre de gestação & 91 & 19,4 & 84 & 92,3 & 7 & 7,7 & \\
\hline Suplementação de vitamina A no pós-parto & & & & & & & 0,269 \\
\hline Sim & 183 & 39,8 & 166 & 90,7 & 17 & 9,3 & \\
\hline Não & 277 & 60,2 & 259 & 93,5 & 18 & 6,5 & \\
\hline Tipo de equipe de saúde da Estratégia Saúde da Fan & & & & & & & 0,427 \\
\hline Programa Mais Médicos & 138 & 29,4 & 125 & 90,6 & 13 & 9,4 & \\
\hline Convencional & 331 & 70,6 & 307 & 92,7 & 24 & 7,3 & \\
\hline \multicolumn{8}{|l|}{ Condições socioeconômicas } \\
\hline \multicolumn{8}{|l|}{ Trabalho materno } \\
\hline Sim & 141 & 30,1 & 132 & 93,6 & 9 & 6,4 & 0,485 \\
\hline Não & 327 & 69,9 & 300 & 91,7 & 27 & 8,3 & \\
\hline \multicolumn{8}{|l|}{ Convivência da mãe com companheiro } \\
\hline Sim & 350 & 74,2 & 320 & 91,4 & 30 & 8,6 & 0,347 \\
\hline Não & 119 & 25,8 & 112 & 94,1 & 7 & 5,9 & \\
\hline \multicolumn{8}{|l|}{ Segurança alimentar e nutricional familiar ${ }^{*}$} \\
\hline Segurança alimentar/insegurança alimentar leve & 387 & 82,5 & 360 & 93,0 & 27 & 7,0 & 0,111 \\
\hline Insegurança alimentar moderada/grave & 82 & 17,5 & 72 & 87,8 & 10 & 12,2 & \\
\hline Classificação socioeconômica $^{\dagger}$ & & & & & & & 0,906 \\
\hline Classes A/B/C & 169 & 36,0 & 156 & 92,3 & 13 & 7,7 & \\
\hline Classes D/E & 300 & 64,0 & 276 & 92,0 & 24 & 8,0 & \\
\hline \multicolumn{8}{|l|}{ Benefício de programas sociais } \\
\hline Frequência da criança à escola de educação infantil & & & & & & & 0,543 \\
\hline Sim & 238 & 50,7 & 221 & 92,9 & 17 & 7,1 & \\
\hline Não & 231 & 49,3 & 211 & 91,3 & 20 & 8,7 & \\
\hline Benefício do Programa Bolsa Família & & & & & & & 0,908 \\
\hline Sim & 346 & 73,8 & 319 & 92,2 & 27 & 7,8 & \\
\hline Não & 123 & 26,2 & 113 & 91,9 & 10 & 8,1 & \\
\hline
\end{tabular}

${ }^{*}$ Com base na Escala Brasileira de Insegurança Alimentar ${ }^{20} .{ }^{\dagger}$ Com base nos critérios da Associação Brasileira de Empresas de Pesquisa ${ }^{21}$. 
Tabela 2. Estado nutricional expresso em escore z do Índice de Massa Corporal-para-idade, de acordo com condições biológicas das mães/crianças, acesso a serviços de saúde, benefício de programas sociais e condições socioeconômicas, de crianças menores de cinco anos residentes em municípios do interior do estado da Paraíba, 2018.

\begin{tabular}{|c|c|c|c|c|c|c|c|}
\hline \multirow{3}{*}{ Variáveis } & \multicolumn{6}{|c|}{$\begin{array}{c}\text { Índice de Massa Corporal-para-idade } \\
\text { (escore } \mathrm{z})\end{array}$} & \multirow{3}{*}{ p-valor } \\
\hline & \multicolumn{2}{|c|}{ Total } & \multicolumn{2}{|c|}{$\leq 2$} & \multicolumn{2}{|c|}{$>2$} & \\
\hline & $\mathbf{n}$ & $\%$ & $\mathbf{n}$ & $\%$ & n & $\%$ & \\
\hline \multicolumn{8}{|l|}{ Condições biológicas das mães/crianças } \\
\hline Idade da criança (anos) & & & & & & & 0,402 \\
\hline$\geq 2$ & 250 & 54,2 & 221 & 88,4 & 29 & 11,6 & \\
\hline$<2$ & 211 & 45,8 & 181 & 85,8 & 30 & 14,2 & \\
\hline Sexo da criança & & & & & & & 0,218 \\
\hline Masculino & 239 & 51,8 & 204 & 85,4 & 35 & 14,6 & \\
\hline Feminino & 222 & 48,1 & 198 & 89,2 & 24 & 10,8 & \\
\hline Idade materna (anos) & & & & & & & 0,659 \\
\hline$\geq 20$ & 412 & 89,8 & 360 & 87,4 & 52 & 12,6 & \\
\hline$<20$ & 47 & 10,2 & 40 & 85,1 & 7 & 14,9 & \\
\hline Estatura da mãe $(\mathrm{cm})$ & & & & & & & 0,115 \\
\hline Adequada & 300 & 65,1 & 267 & 89,0 & 33 & 11,0 & \\
\hline Inadequada $(<155)$ & 161 & 34,9 & 135 & 83,9 & 26 & 16,1 & \\
\hline Índice de Massa Corporal da mãe $\left(\mathrm{Kg} / \mathrm{m}^{2}\right)$ & & & & & & & 0,058 \\
\hline Adequado & 185 & 40,1 & 168 & 90,8 & 17 & 9,2 & \\
\hline Sobrepeso e obesidade $(\geq 25)$ & 276 & 59,9 & 234 & 84,8 & 42 & 15,2 & \\
\hline \multicolumn{8}{|l|}{ Acesso a serviços de saúde } \\
\hline Trimestre de início do pré-natal & & & & & & & 0,023 \\
\hline $1^{\circ}$ trimestre de gestação & 370 & 80,4 & 329 & 88,9 & 41 & 11,1 & \\
\hline Após $1^{\circ}$ trimestre de gestação & 90 & 19,6 & 72 & 80,0 & 18 & 20,0 & \\
\hline Suplementação de vitamina A no pós-parto & & & & & & & 0,452 \\
\hline Sim & 179 & 39,6 & 153 & 85,5 & 26 & 14,5 & \\
\hline Não & 273 & 60,4 & 240 & 87,9 & 33 & 12,1 & \\
\hline Tipo de equipe de saúde da Estratégia Saúde da Família & & & & & & & 0,281 \\
\hline Programa Mais Médicos & 137 & 29,7 & 123 & 89,8 & 14 & 10,2 & \\
\hline Convencional & 324 & 70,3 & 279 & 86,1 & 45 & 13,9 & \\
\hline \multicolumn{8}{|l|}{ Condições socioeconômicas } \\
\hline Trabalho materno & & & & & & & 0,772 \\
\hline Sim & 140 & 30,4 & 123 & 87,9 & 17 & 12,1 & \\
\hline Não & 320 & 69,6 & 278 & 86,9 & 42 & 13,1 & \\
\hline Convivência da mãe com companheiro & & & & & & & 0,544 \\
\hline Sim & 343 & 74,4 & 301 & 87,8 & 42 & 12,2 & \\
\hline Não & 118 & 25,6 & 101 & 85,6 & 17 & 14,4 & \\
\hline Segurança alimentar e nutricional familiar ${ }^{*}$ & & & & & & & 0,250 \\
\hline Segurança alimentar/insegurança alimentar leve & 382 & 82,9 & 330 & 86,4 & 52 & 13,6 & \\
\hline Insegurança alimentar moderada/grave & 79 & 17,1 & 72 & 91,1 & 7 & 8,9 & \\
\hline Classificação socioeconômica $^{\dagger}$ & & & & & & & 0,447 \\
\hline Classes A/B/C & 169 & 36,7 & 150 & 88,8 & 19 & 11,2 & \\
\hline Classes D/E & 292 & 63,3 & 252 & 86,3 & 40 & 13,7 & \\
\hline \multicolumn{8}{|l|}{ Benefício de programas sociais } \\
\hline Frequência da criança à escola de educação infantil & & & & & & & 0,515 \\
\hline Sim & 237 & 51,4 & 209 & 88,2 & 28 & 11,8 & \\
\hline Não & 224 & 48,6 & 193 & 86,2 & 31 & 13,8 & \\
\hline Benefício do Programa Bolsa Família & & & & & & & 0,049 \\
\hline Sim & 338 & 73,3 & 301 & 89,1 & 37 & 10,9 & \\
\hline Não & 123 & 26,7 & 101 & 82,1 & 22 & 17,9 & \\
\hline
\end{tabular}

${ }^{*}$ Com base na Escala Brasileira de Insegurança Alimentar ${ }^{20} .{ }^{\dagger}$ Com base nos critérios da Associação Brasileira de Empresas de Pesquisa ${ }^{21}$. 
Estatura/idade (escore $\mathrm{z}$ )

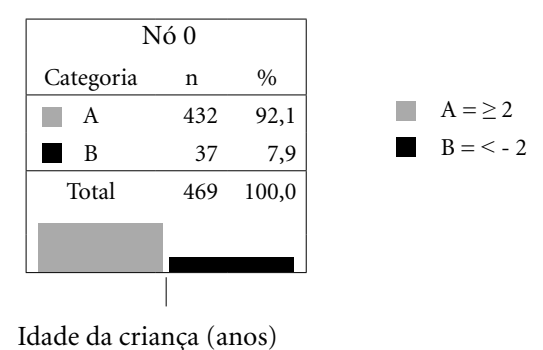

Valor $\mathrm{p}$ ajustado $=0,018 ;$ qui-quadrado $=5,588 ; \mathrm{df}=1$
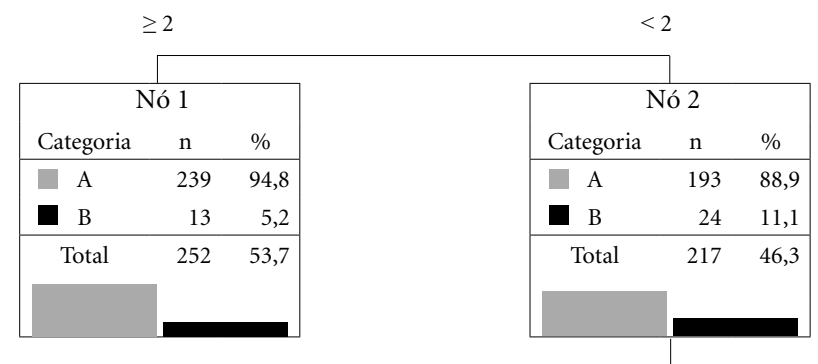

Segurança alimentar e nutricional familiar

Valor $\mathrm{p}$ ajustado $=0,018 ;$ qui-quadrado $=6,980 ; \mathrm{df}=1$
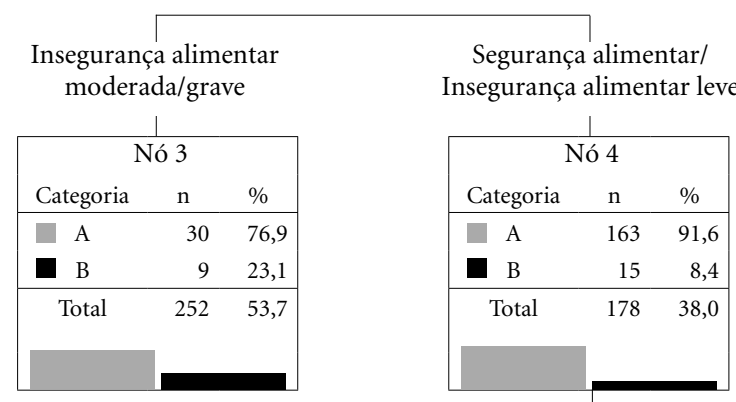
Insegurança alimentar leve

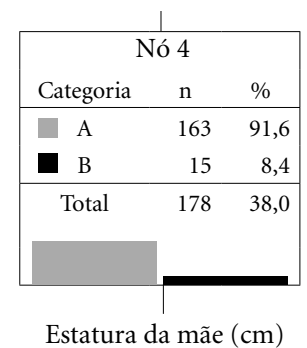

Valor $\mathrm{p}$ ajustado $=0,028 ;$ qui-quadrado $=4,814 ; \mathrm{df}=1$

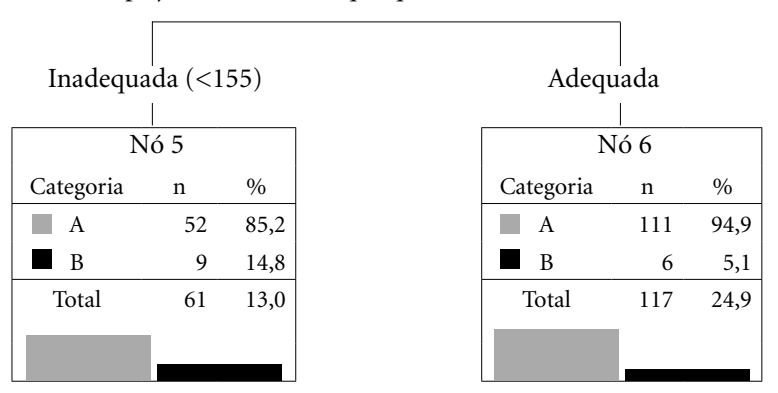

Figura 3. Análise multivariada por meio da árvore de decisão para o déficit de estatura, ajustada pelos fatores investigados, em crianças menores de cinco anos residentes em municípios do interior do estado da Paraíba, 2018.

Df: degrees of freedom.

Fonte: Autores. 
Índice de Massa Corporal/idade (escore z)

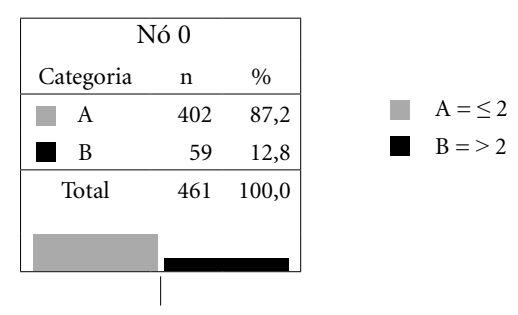

Benefício do Programa Bolsa Família

Valor $\mathrm{p}$ ajustado $=0,049 ;$ qui-quadrado $=3,891 ; \mathrm{df}=1$
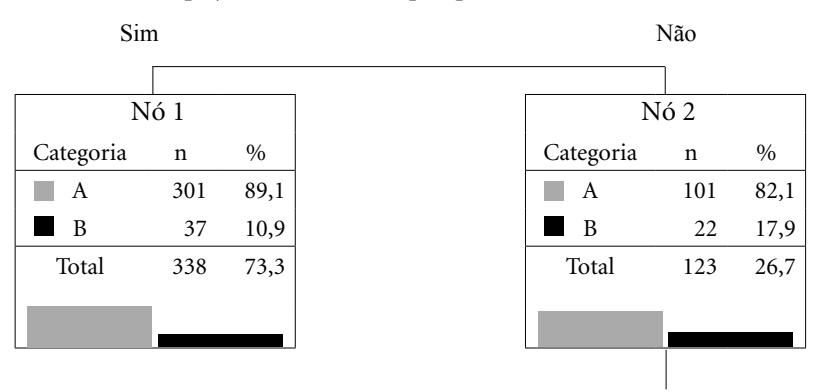

Classificação socioeconômica

Valor $\mathrm{p}$ ajustado $=0,006$; qui-quadrado $=7,414 ; \mathrm{df}=1$

Classes $\mathrm{A} / \mathrm{B} / \mathrm{C}$

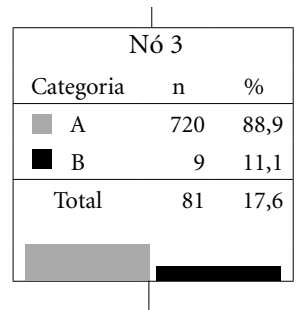

Índice de Massa Corporal da mãe $(\mathrm{kg} / \mathrm{m} 2)$ Valor $\mathrm{p}$ ajustado $=0,027 ;$ qui-quadrado $=4,876 ; \mathrm{df}=1$

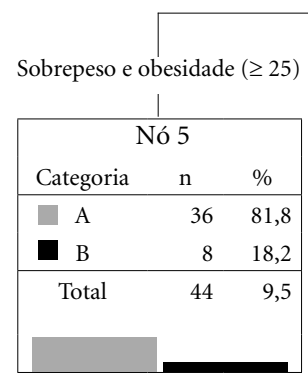

Classes D/E

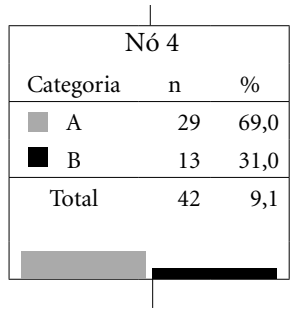

Estatura da mãe $(\mathrm{cm})$ Valor $\mathrm{p}$ ajustado $=0,019 ;$ qui-quadrado $=5,469 ; \mathrm{df}=1$
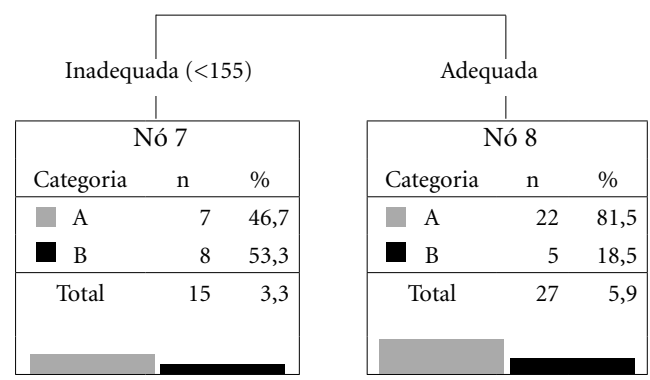

Figura 4. Análise multivariada por meio da árvore de decisão para o excesso de peso (sobrepeso e obesidade), ajustada pelos fatores investigados, em crianças menores de cinco anos residentes em municípios do interior do estado da Paraíba, 2018.

Df: degrees of freedom.

Fonte: Autores. 
Neste estudo, ser menor de dois anos representou a exposição mais próxima do déficit de estatura, o que respalda os achados divulgados na literatura ${ }^{27,28}$. Desse modo, é importante reforçar a necessidade de investimentos em medidas preventivas, como a garantia de saneamento ambiental, a suplementação durante a gravidez e da criança e a promoção do aleitamento materno exclusivo e da alimentação complementar de qualidade ${ }^{29}$.

O comprometimento do crescimento linear associado à IAN $^{30-32}$, inclusive entre menores de dois anos ${ }^{31}$, também foi observado no atual estudo. $\mathrm{O}$ modo como a IAN influencia o crescimento da criança reflete, principalmente, o déficit qualitativo e quantitativo da alimentação ${ }^{31}$. Essas hipóteses se revestem de significados relevantes com base nos resultados obtidos na Pesquisa de Orçamentos Familiares, por meio da qual foi possível observar maior gravidade da IAN em domicílios com menor aquisição alimentar per capita, inclusive para grupos de alimentos saudáveis ${ }^{33}$.

A importância dos antecedentes nutricionais da gestação e o compartilhamento de fatores genéticos, ambientais e socioeconômicos por parte da díade mãe-filho justificam a influência da estatura materna no potencial de crescimento de seus filhos ${ }^{2,13}$. A observação das crianças desta pesquisa permitiu confirmar achados concernentes a essa relação obtidos em estudos prévios ${ }^{2,6,13,27}$.

Segundo o modelo da árvore de decisão do excesso de peso, esse desfecho foi mais prevalente nas crianças não beneficiárias do PBF. Embora esse resultado coincida com o observado em estudos de base nacional ${ }^{34,35}$, revisões sistemáticas não são conclusivas em relação ao assunto ${ }^{15,36,37}$. Os efeitos positivos do Programa no estado nutricional da criança podem derivar de melhorias na quantidade e na qualidade da alimentação, assim como de conhecimentos sobre alimentação e do acompanhamento da saúde da criança em decorrência das condicionalidades de saúde ${ }^{35-37}$. Além disso, sugere-se que, nas crianças brasileiras, o peso excessivo atinja principalmente aquelas de melhor situação social, característico de países onde o aumento dos níveis de obesidade ainda é recente ${ }^{34}$.

A relação entre o nível socioeconômico e o excesso de peso infantil, sobretudo nos países de renda média ou baixa, é controversa e complexa, com mecanismos ainda não esclarecidos que distinguem uma multiplicidade de determinantes $^{34,38}$. Quando o risco de excesso de peso é maior em condições sociais de vulnerabilidade, como no estudo em questão para não beneficiários do PBF de classes socioeconômicas inferiores, cogita-se a influência do acesso limitado à alimentação saudável e da falta de conhecimento sobre os danos relacionados ao problema ${ }^{5,13,38}$. Nessa linha de raciocínio, estudos longitudinais mostraram que o risco de obesidade associado à pobreza pode ser mitigado com melhorias no status econômico capazes de favorecer os comportamentos alimentares ${ }^{39,40}$.

A associação da estatura materna com o excesso de peso da criança pode estar relacionada a mecanismos de adaptação, como catch-up (ganhos maiores do que os da normalidade) e distúrbios hormonais, resultantes de condições do período pré-natal e pós-natal desfavoráveis - a exemplo do nascimento com baixo peso decorrente da estatura materna deficiente - que predispõem ao ganho de peso excessivo ${ }^{41,42}$. Embora não seja possível precisar a trajetória do catch-up nas crianças desse estudo, presume-se que o fenômeno tenha sido mais forte naquelas de classes socioeconômicas inferiores não beneficiárias do PBF, que foram as que apresentaram maiores prevalências de excesso de peso, assemelhandose aos achados registrados em estudo de coorte ${ }^{43}$.

O excesso de peso materno como fator de risco para sobrepeso e obesidade da criança reproduz a interação multinível de determinantes biológicos, comportamentais e sociais, que desde os estágios iniciais da vida (fase pré-concepcional, pré-natal e do nascimento aos dois anos) modelam a predisposição de transferência do status de peso entre gerações ${ }^{2,38,44}$. Nesse processo, a influência do ambiente obesogênico, como alimentação não saudável e sedentarismo, que levam a adaptações metabólicas precursoras de obesidade e à hereditariedade dessa condição, respalda a relevância de medidas preventivas com foco na promoção de práticas alimentares saudáveis e do peso ideal ${ }^{2,12,38}$, aplicáveis neste estudo para as crianças de melhor situação socioeconômica não beneficiárias do PBF nas quais os resultados registrados foram harmônicos.

Esse estudo tem limitações, uma vez que seu delineamento transversal não permite estabelecer a temporalidade das associações analisadas. Além disso, não foram incluídos alguns fatores que interferem no estado nutricional das crianças menores de cinco anos, como a idade gestacional ao nascimento, o peso ao nascer, as práticas de cuidado, o consumo alimentar e a situação de saúde.

Do ponto de vista metodológico, deve ser ressaltado que este estudo mostra novas perspectivas de análises para a compreensão da baixa estatura 
e do sobrepeso e obesidade em crianças. Assim, contribui para o avanço do conhecimento ao delimitar exposições que podem ser relevantes para grupos específicos de indivíduos com características semelhantes. A capacidade analítica da árvore de decisão pode ser sublinhada ao permitir identificar associações não predeterminadas nas análises bivariadas, o que traduz, possivelmente, a interação complexa dos fatores estudados.

\section{Conclusões}

Os resultados do presente estudo revelam prevalências expressivas de déficit de estatura e de excesso de peso em crianças menores de cinco anos usuárias da ESF, as quais se relacionam ao estado nutricional materno. Embora alguns mecanismos e vias dessa transferência intergeracional ainda não estejam claros, sugere-se a existência de relações mediadas pelo compartilhamento de características biológicas e socioambientais.

Verificou-se, ainda, diferenças nos determinantes dos desvios nutricionais de acordo com certas características. A idade da criança e a SAN se sobressaíram por sua repercussão na baixa estatura. Para o excesso de peso, o PBF e a situação socioeconômica representaram as variáveis mais relevantes.

Esses achados fornecem subsídios para a orientação de estratégias multisetoriais e a elaboração de ações nos municípios direcionadas à prevenção e ao controle de tais problemas. Para esses fins, deve se destacar a importância dos cuidados oferecidos às mães e às crianças, incluindo medidas de saúde pública eficazes que possam assegurar benefícios à saúde tanto de forma imediata quanto a longo prazo.

\section{Colaboradores}

MM Oliveira, EES Santos e IM Bernardino participaram da análise e interpretação dos dados, da elaboração do rascunho, revisão crítica do conteúdo e aprovação da versão final do manuscrito. D Figueroa Pedraza participou da concepção e planejamento, análise e interpretação dos dados, elaboração do rascunho, revisão crítica do conteúdo e aprovação da versão final do manuscrito. 


\section{Referências}

1. Mia MN, Rahman MS, Roy PK. Sociodemographic and geographical inequalities in under and overnutrition among children and mothers in Bangladesh: a spatial modelling approach to a nationally representative survey. Public Health Nut 2018; 21(13):2471-2481.

2. Felisbino-Mendes MS, Villamor E, Velasquez-Melendez G. Association of Maternal and Child Nutritional Status in Brazil: A Population Based Cross-Sectional Study. PLoS ONE 2014; 9(1):e87486.

3. World Health Organization (WHO). Global nutrition targets 2025: policy brief series. Geneva: WHO; 2014.

4. Organização das Nações Unidas no Brasil (ONU Brasil). Fome zero e agricultura sustentável. Brasília: ONU Brasil; 2017.

5. Pereira IFS, Andrade LMB, Spyrides MHC, Lyra CO. Estado nutricional de menores de 5 anos de idade no Brasil: evidências da polarização epidemiológica nutricional. Cien Saude Colet 2017; 22(10):3341-3352.

6. Rachmi CN, Agho KE, Li M, Baur LA. Stunting, Underweight and Overweight in Children Aged 2.0-4.9 Years in Indonesia: Prevalence Trends and Associated Risk Factors. PLoS ONE 2016; 11(5):e0154756.

7. Brasil. Ministério da Saúde (MS). Vigitel Brasil 2019: vigilância de fatores de risco e proteção para doenças crônicas por inquérito telefônico: estimativas sobre frequência e distribuição sociodemográfica de fatores de risco e proteção para doenças crônicas nas capitais dos 26 estados brasileiros e no Distrito Federal em 2019. Brasília: MS; 2020.

8. Finucane MM, Stevens GA, Cowan MJ, Danaei G, Lin JK, Paciorek CJ, Singh GM, Gutierrez HR, Lu Y, Bahalim AN, Farzadfar F, Riley LM, Ezzati M. National, regional, and global trends in body mass index since 1980: systematic analysis of health examination surveys and epidemiological studies with 960 country-years and 9.1 million participants. Lancet 2011; 377(9765):557-567.

9. Brasil. Ministério da Saúde (MS). Pesquisa Nacional de Demografia e Saúde da Criança e da Mulher - PNDS 2006: dimensões do processo reprodutivo e da saúde da criança. Brasília: MS; 2009.

10. United Nations Children's Fund, World Health Organization (WHO), World Bank Group. Joint child malnutrition estimates - levels and trends. Washington: WHO; 2018.

11. Akombi BJ, Agho KE, Hall JJ, Wali N, Renzaho AMN. Stunting, wasting and underweight in Sub-Saharan Africa: a systematic review. Int J Environ Res Public Health 2017; 4(8):863.

12. Wang Y, Min J, Khuri J, Li M. A Systematic examination of the association between parental and child obesity across countries. Adv Nutr 2017; 8(3):436-448.

13. Figueroa Pedraza D. Preditores de riscos nutricionais de crianças assistidas em creches em município de porte médio do Brasil. Cad Saude Colet 2017; 25(1):14-23

14. Sousa CPC, Olinda RA, Figueroa Pedraza D. Prevalence of stunting and overweight/obesity among Brazilian children according to different epidemiological scenarios: systematic review and meta-analysis. Sao Paulo Med J 2016; 34(3):251-262.
15. Martins APB, Canella DS, Baraldi LG, Monteiro CA. Transferência de renda no Brasil e desfechos nutricionais: revisão sistemática. Rev Saude Publ 2013; 47(6):1159-1171.

16. Kemper ES, Mendonça AVM, Sousa MF. Programa Mais Médicos: panorama da produção científica. Cien Saude Colet 2016; 21(9):2785-2796.

17. Brasil. Portaria $\mathrm{n}^{\circ} 2.706$, de 18 de outubro de 2017 Lista os Municípios que finalizaram a adesão ao Programa Saúde na Escola para o ciclo 2017/2018 e os habilita ao recebimento do teto de recursos financeiros pactuados em Termo de Compromisso e repassa recursos financeiros para Municípios prioritários para ações de prevenção da obesidade infantil com escolares. Diário Oficial da União 2017; 20 out.

18. Brasil. Ministério da Saúde (MS). Orientações para a coleta e análise de dados antropométricos em serviços de saúde: Norma Técnica do Sistema de Vigilância Alimentar e Nutricional - SISVAN. Brasília: MS; 2011.

19. Onis M, Onyango AW, Van den Broeck J, Chumlea WC, Martorell R. Measurement and standardization protocols for anthropometry used in the construction of a new international growth reference. Food Nutr Bull 2004; 25(Supl. 1):15-27.

20. Segall-Corrêa AM, Marin-León L, Melgar-Quiñonez $\mathrm{H}$, Pérez-Escamilla R. Refinement of the Brazilian Household Food Insecurity Measurement Scale: Recommendation for a 14-item EBIA. Rev Nutr 2014; 27(2):241-251.

21. Brasil. Associação Brasileira de Empresas de Pesquisa (ABEP). Critério de classificação econômica Brasil. São Paulo: ABEP; 2016.

22. Bernardino IM, Barbosa KGN, Nóbrega LM, Cavalcante GMS, Ferreira EF, D’Avila S. Violência contra mulheres em diferentes estágios do ciclo de vida no Brasil: um estudo exploratório. Rev Bras Epidemiol 2016; 19(4):740-752.

23. Soárez PC, Soares MO, Novaes HMD. Modelos de decisão para avaliações econômicas de tecnologias em saúde. Cien Saude Colet 2014; 19(10):4209-4222.

24. Figueroa-Pedraza D. Implementation of food and nutrition actions in the context of family health strategy, Paraíba, Brazil. Rev Fac Nac Salud Pública 2019; 37(3):98-109.

25. Rivera JA, Pedraza LS, Martorell R, Gil A. Introduction to the double burden of undernutrition and excess weight in Latin America. Am J Clin Nutr 2014; 100(6):1613S-1616S.

26. Conde WL, Monteiro CA. Nutrition transition and double burden of undernutrition and excess of weight in Brazil. Am J Clin Nutr 2014; 100(6):1617S-1622S.

27. Abdulahi A, Shab-Bidar S, Rezaei S, Djafarian K. Nutritional Status of Under Five Children in Ethiopia: A Systematic Review and Meta-Analysis. Ethiop J Health Sci 2017; 27(2):175-188.

28. Figueroa Pedraza D, Souza MM, Rocha ACD. Fatores associados ao estado nutricional de crianças pré-escolares brasileiras assistidas em creches públicas: uma revisão sistemática. Rev Nutr 2015; 28(4):451-464.

29. Cunha AJ, Leite AJ, Almeida IS. The pediatrician's role in the first thousand days of the child: the pursuit of healthy nutrition and development. J Pediatr 2015; 6(1):44-51. 
30. Gubert MB, Spaniol AM, Bortolini GA, Pérez-Escamilla R. Household food insecurity, nutritional status and morbidity in Brazilian children. Public Health Nutr 2016; 19(12):2240-2245.

31. Monteiro F, Schmidt ST, Costa IB, Almeida CCB, Matuda NS. Bolsa Família: insegurança alimentar e nutricional de crianças menores de cinco anos. Cien Saude Colet 2014; 19(5):1347-1357.

32. Betebo B, Ejajo T. Alemseged F, Massa D. Household Food Insecurity and its association with nutritional status of children 6-59 months of age in East Badawacho District, South Ethiopia. J Environ Public Health 2017; 2017:6373595.

33. Brasil. Instituto Brasileiro de Geografia e Estatística (IBGE). Pesquisa de Orçamentos Familiares 2017-2018: análise da segurança alimentar no Brasil. Rio de Janeiro: IBGE; 2020.

34. Santos LP, Gigante DP. Relação entre insegurança alimentar e estado nutricional de crianças brasileiras menores de cinco anos. Rev Bras Epidemiol 2013; 16(4):984-994

35. Pinho Neto VR, Berriel CM. Transferências condicionais de renda e nutrição: efeitos do bolsa família nas áreas rurais e urbanas do Brasil. Econ Aplic 2017; 21(2):185-205.

36. Gamboa-Delgado EM, Cossío TG, Colchero-Aragonés A. Riesgo de sobrepeso en niños preescolares beneficiarios de programas de ayuda alimentaria. Rev Salud Pública 2016; 18(4):643-655.

37. Groot R, Palermo T, Handa S, Ragno LP, Peterman A. Cash transfers and child nutrition: pathways and impacts. Dev Policy Rev 2017; 35:621-43.

38. Haire-Joshu D, Tabak R. Preventing obesity across generations: evidence for early life intervention. Annu Rev Public Health 2017; 37:253-271.

39. Oddo VM, Jones-Smith JC. Gains in income during early childhood are associated with decreases in BMI $z$ scores among children in the United States. Am J Clin Nutr 2015; 101(6):1225-1231.
40. Goisis A, Sacker A, Kelly Y. Why are poorer children at higher risk of obesity and overweight? A UK cohort study. Eur J Public Health 2016; 26(1):7-13.

41. Ribeiro AM, Lima MC, Lira PIC, Silva GAP. Low birth weight and obesity: causal or casual casual association? Rev Paul Pediatr 2015; 33(3):340-348.

42. Kuhn-Santos RC, Suano-Souza FI, Puccini RF, Strufaldi MWL. Fatores associados ao excesso de peso e baixa estatura em escolares nascidos com baixo peso. Cien Saude Colet 2019; 24(2):361-370.

43. Kiy AM, Rugolo LMSS, De Luca AKC, Corrente JE. Growth of preterm low birth weight infants until 24 months corrected age: effect of maternal hypertension. J Pediatr 2015; 91(3):256-262.

44. Tchoubi S, Sobngwi-Tambekou J, Noubiap JJ, Asangbeh SL, Nkoum BA, Sobngwi E. Prevalence and risk factors of overweight and obesity among children aged 6-59 months in Cameroon: a multistage, stratified cluster sampling nationwide survey. PLoS ONE 2015; 10(12):e0143215.

Artigo apresentado em 07/12/2019

Aprovado em 22/01/2021

Versão final apresentada em 24/01/2021

Editores-chefes: Romeu Gomes, Antônio Augusto Moura da Silva 